\title{
Evidence for the synthesis and release of a glycoprotein by mouse blastocysts
}

\author{
S. B. Fishel and M. A. H. Surani* \\ Marshall Laboratory, Physiological Laboratory, University of Cambridge, \\ Cambridge CB2 3EG, U.K.
}

\begin{abstract}
Summary. Mouse blastocysts were incubated in microdrop cultures in the presence of $\mathrm{D}-\left[6-{ }^{3} \mathrm{H}\right]$ glucosamine. The embryos and the incubation medium were analysed for the incorporation of label into acid-precipitable material. Of the precursor incorporated into blastocysts, $8-13 \%$ was detected in the medium. Several glycoproteins were found in the blastocysts and one of molecular weight about 87000 was also detected in the extracellular medium. In the presence of tunicamycin, the amount of the labelled glycoprotein was reduced by $30-40 \%$ in the embryos and $47-69 \%$ in the medium. However, the same relative proportion of label was still detected in the extracellular fraction of $87000 \mathrm{~mol}$. wt. The study demonstrates the synthesis and release of a glycoprotein by mouse blastocysts, the mechanism of which is tunicamycin-insensitive.
\end{abstract}

\section{Introduction}

Amongst several aspects of blastocyst-uterine interactions involved in the initiation and synchronization of blastocyst implantation, one of the interesting features of the early embryo-uterine relationship is the production of extracellular macromolecules and hormones by preimplantation blastocysts (Heap, Flint \& Gadsby, 1979). Such molecules could induce a localized effect on the uterus which may be necessary for implantation and/or ensure the maintenance of early pregnancy. There is increasing evidence that blastocysts synthesize and secrete steroids and macromolecules (Knobil, 1973; Perry, Heap \& Amoroso, 1973; Wiley, 1974; Dickmann, Dey \& Sengupta, 1976). Synthesis of glycoproteins, especially gonadotrophins, by early embryos is thought to occur in several species, including man (Saxena, Hasan, Haour \& Schmidt-Gollwitzer, 1974; Beling, Cederqvist \& Fuchs, 1976), and these hormone-like substances may ensure the maintenance of early pregnancy by their direct or indirect effect via the uterus, or by preventing the regression of the corpus luteum. Other localized responses, such as induction of specific uterine proteins and increase in mitotic activity, occur in the presence of blastocysts, perhaps partly in response to the release of macromolecules by blastocysts; embryos at a stage earlier than the blastocyst do not evoke many of these responses.

Ultrastructural examination of preimplantation embryos shows that by the blastocyst stage the rough endoplasmic reticulum is well established and these embryos could therefore synthesize membrane-bound and secretory glycoproteins (Enders, 1971). Mouse blastocysts are known to synthesize numerous proteins and glycoproteins but attempts to detect synthesis and release of macromolecules has so far been unsuccessful (Van Blerkom, Chavez \& Bell, 1979).

* Present address: A.R.C. Institute of Animal Physiology, Animal Research Station, 307 Huntingdon Road, Cambridge CB3 0JQ, U.K. 
This problem was examined in the present study of mouse blastocysts in microdrop cultures in vitro, in the presence or absence of the drug tunicamycin. Tunicamycin primarily inhibits the synthesis of $\mathrm{N}$-acetylglucosaminyl phophosphoryl dolichol phosphate, and has been recently studied with mouse blastocysts (Surani, 1979).

\section{Materials and Methods}

Blastocysts were retrieved from uterine horns of CFLP mice (Anglia Laboratories) at 10:00 h on Day 4 of pregnancy (Day $1=$ day of vaginal plug), using $0.1 \mathrm{M}$-phosphate-buffered saline (PBS) with $4 \mathrm{mg}$ bovine serum albumin $/ \mathrm{ml}, \mathrm{pH} 7.2$ at $37^{\circ} \mathrm{C}$. The embryos were washed twice and divided into control (C) and experimental (E) groups. Four replicate experiments were done. The embryos in both groups were cultured for $6 \mathrm{~h}$ in an embryo culture medium (see Surani, 1979) with $10 \%$ fetal calf serum in plastic Petri dishes (Falcon). The medium for Group E blastocysts contained $1 \mu \mathrm{g}$ tunicamycin $/ \mathrm{ml}$ dissolved in dimethylsulphoxide whilst the vehicle alone was added to Group $\mathrm{C}$ cultures. The blastocysts, 70-100/group, were then removed and washed 6 times to remove serum and glucose and cultured together in a $5 \mu \mathrm{l}$ drop of glucose-free medium with pyruvate as an alternative energy substrate and $2.5 \mu \mathrm{Ci} \mathrm{D}-\left[6-{ }^{3} \mathrm{H}\right]$ glucosamine (sp. act. $38 \mathrm{Ci} / \mathrm{mmol}$; Radiochemical Centre, Amersham); embryos in Group $\mathrm{E}$ were cultured continuously in the presence of tunicamycin. After incubation for $9 \mathrm{~h}$ the $5 \mu \mathrm{l}$ drop of culture medium surrounding the blastocysts was aspirated by means of a finely drawn Pasteur pipette (inner bore $<50 \mu \mathrm{m}$ ) and then added to $95 \mu \mathrm{l} 0.01 \mathrm{M}$-PBS containing $0.1 \%$ sodium dodecyl sulphate and $0.14 \mathrm{M}$-2-mercaptoethanol (lysis buffer) before immediate storage at $-20^{\circ} \mathrm{C}$. The embryos were removed from the Petri dishes, washed 6 times with PBS containing $10 \mathrm{mg}$ polyvinylpyrrolidone $/ \mathrm{ml}$ and then added to $100 \mu \mathrm{l}$ lysis buffer for storage at $-20^{\circ} \mathrm{C}$. Within 5 days all samples were thawed and heated at $65^{\circ} \mathrm{C}$ for $1 \mathrm{~h}$. Quadruplicate aliquots of $2.5 \mu \mathrm{l}$ were removed from each sample and deposited on GF/A glass-fibre discs for quantitative estimation of incorporation of the precursor after precipitation of proteins by cold $10 \%(\mathrm{w} / \mathrm{v})$ trichloroacetic acid. The remainder of the sample $(90 \mu \mathrm{l})$ from each group was used for qualitative analysis on $6 \%$ polyacrylamide-sodium dodecyl sulphate gels.

\section{Results}

As shown in Table 1, the amount of labelled glycoproteins released from blastocysts into the medium was approximately $8-13 \%$ of the total precursor incorporated in the blastocyst. The incorporation of $\left[{ }^{3} \mathrm{H}\right]$ glucosamine into blastocysts in Group $\mathrm{E}$ was reduced by $30-40 \%$ and the amount of labelled glycoproteins released in the medium by $47-69 \%$ when compared with the incorporation of the precursor in Group C blastocysts. However, even in this group, labelled glycoproteins were released by blastocysts into the extracellular medium and this value was in the region of $4-5 \%$ of the total precursor incorporated in the blastocyst.

Several glycopeptides labelled with $\left[{ }^{3} \mathrm{H}\right]$ glucosamine were detected in the blastocysts in Group $\mathrm{C}$ and there was substantial incorporation of the precursor (approximately $35 \%$ of the total amino sugar incorporated) in the first few fractions (Text-fig. 1). However, when the extracellular medium was analysed for the labelled glycopeptides released by blastocysts, the incorporation in these first few fractions amounted to about $8 \%$ of the total radioactivity of the sample. The predominant peak was observed with approximate molecular weight of 87000 and this represented about $28 \%$ of the total acid-precipitable material in the extracellular medium. In Group E blastocysts treated with tunicamycin, the incorporation of the precursor in the majority of the fractions was substantially inhibited except for the major fraction near the top of the gel. As demonstrated previously (Surani, 1979), glycosylation of most of the fractions was markedly 


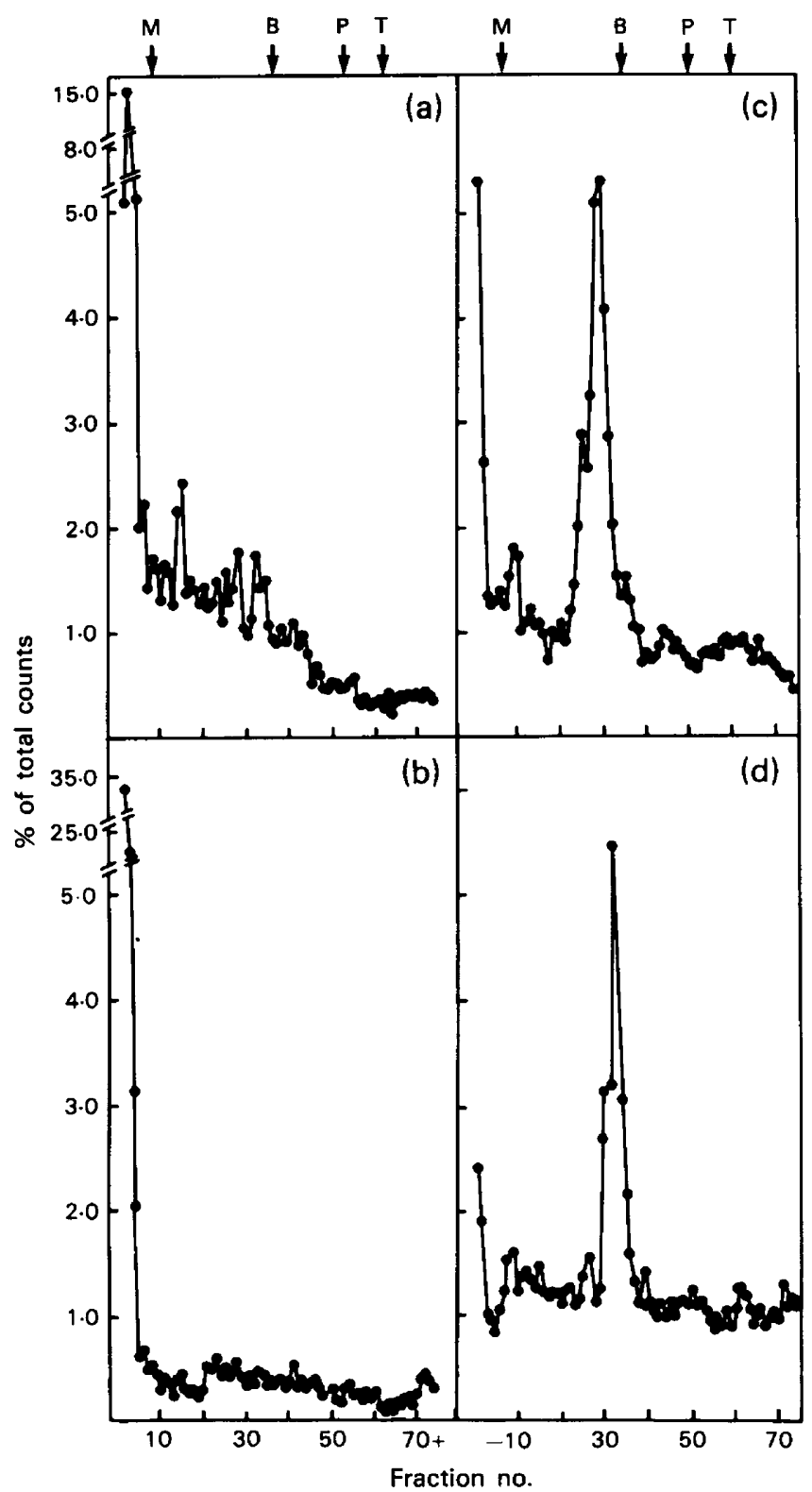

Text-fig. 1. Qualitative analysis of the incorporation of $\left[{ }^{3} \mathrm{H}\right]$ glucosamine into (a) control blastocysts, (b) experimental blastocysts, (c) control extracellular medium and (d) experimental extracellular medium. The labelled glycoproteins in the samples $(90 \mu \mathrm{l})$ were analysed on $6 \%$ polyacrylamide-sodium dodecyl sulphate gels. The total radioactivities were (a) $9.1 \times 10^{3}-13.2$ $\times 10^{3}$ c.p.m. (b) $2.5 \times 10^{3}-3.2 \times 10^{3}$ c.p.m. (c) $1.2 \times 10^{3}-1.7 \times 10^{3}$ c.p.m. and (d) $0.35 \times$ $10^{3}-0.47 \times 10^{3}-0.47 \times 10^{3}$ c.p.m. The gels were calibrated using myosin (M: mol. wt 220000$)$ bovine serum albumin (B: mol. wt 66500 ), peroxidase (P: mol. wt 40000 ) and trypsin (T: mol. wt 23000 ). 
Table 1 . Synthesis and release of glycoproteins from mouse blastocysts labelled with $\left[{ }^{3} \mathrm{H}\right]$ glucosamine and in the absence (controls) or presence (experimental) of

$1 \mu \mathrm{g}$ tunicamycin/ml

\begin{tabular}{|c|c|c|c|c|}
\hline & Exp. 1 & Exp. 2 & Exp. 3 & Exp. 4 \\
\hline \multicolumn{5}{|l|}{ Control blastocysts } \\
\hline No. & 99 & 80 & $7:$ & 76 \\
\hline \multicolumn{5}{|l|}{ Incorporation } \\
\hline Into blastocysts & 5550 & 4085 & 5280 & 5985 \\
\hline Into medium & 561 & 389 & 663 & 401 \\
\hline \multicolumn{3}{|l|}{ Labelled glycoproteins released } & 12.56 & 8.04 \\
\hline \multicolumn{5}{|l|}{ Experimental blastocysts } \\
\hline $\begin{array}{l}\text { No. } \\
\text { Incorporation }\end{array}$ & 102 & 76 & 93 & 104 \\
\hline $\begin{array}{l}\text { Into blastocysts (\% inhibition } \\
\text { compared with controls) }\end{array}$ & $\begin{array}{l}3603 \\
(35.08)\end{array}$ & $\begin{array}{l}2625 \\
(35.74)\end{array}$ & $\begin{array}{l}3726 \\
(29 \cdot 43)\end{array}$ & $\begin{array}{l}2934 \\
(41 \cdot 14)\end{array}$ \\
\hline $\begin{array}{l}\text { Into medium (\% inhibition } \\
\text { compared with controls) }\end{array}$ & $\begin{array}{l}182 \\
(50.05)\end{array}$ & $\begin{array}{l}96 \\
(61.55)\end{array}$ & $\begin{array}{l}146 \\
(68 \cdot 79)\end{array}$ & $\begin{array}{l}125 \\
(47 \cdot 01)\end{array}$ \\
\hline $\begin{array}{l}\text { Labelled glycoproteins released } \\
\text { from blastocysts }(\%)\end{array}$ & $5 \cdot 05$ & 3.66 & 3.92 & $4 \cdot 26$ \\
\hline
\end{tabular}
for $9 \mathrm{~h}$.

The incorporation values are calculated in terms of c.p.m./blastocyst after incubation

inhibited by tunicamycin (resulting in the low incorporation of $\left[{ }^{3} \mathrm{H}\right]$ glucosamine). There was also a marked reduction in the overall amount of labelled glycoproteins released by Group $\mathrm{E}$ blastocysts into the medium compared with that released by Group $\mathrm{C}$ blastocysts, and in the proportion of the incorporation into Group E blastocysts. However, the relative distribution of the precursor in the extraembryonic glycoproteins was essentially unchanged since up to $26 \%$ of the total incorporation was still detected in the fraction of apparent molecular weight of 87000 .

\section{Discussion}

Previous study (Surani, 1979) using experimental procedures similar to those described above showed that tunicamycin reversibly inhibits glycosylation of glycoproteins linked with an $N$ glycoside without having a marked effect on overall protein synthesis in mouse blastocysts; the incorporation of mannose and glucosamine is substantially reduced in the majority of the glycopeptides in absolute terms as well as in the relative distribution of the incorporated precursor in the separated glycopeptides detected in polyacrylamide gels (Surani, 1979). Although the overall release of labelled glycoproteins from blastocysts is reduced in the presence of tunicamycin, the relative distribution of the precursor in the majority of the glycopeptide fractions separated on the gels is essentially similar to that in the control group. The reduction in the amount of labelled glycoproteins could result from some non-specific effect of the antibiotic. The glycosylation of the major component detected in the culture medium may not, therefore, occur via the dolichol-mediated pathway. Alternatively, the component of molecular weight 87000 could be a modified fragment of the large molecular weight component detected near the top of the gel which is also insensitive to inhibition by tunicamycin.

This study demonstrates, for the first time, synthesis and release of a major glycoprotein by preimplantation mouse blastocyst. This macromolecule may be a specific secretory product of embryos that may act as a signal important for the embryo-maternal relationship before and during the initiation of blastocyst implantation (Heap et al., 1979). Its action could include induction of a local uterine response, a direct or indirect influence on the corpus luteum or a means to neutralize immunological rejection of the implanting embryo by the mother (Amoroso 
\& Perry, 1975; Siiteri et al., 1977; Beer \& Billingham, 1979). This component might, however, be a cell surface glycoprotein of preimplantation embryos which is subsequently shed in its original or modified form.

We thank Dr S. Kimber, Mrs S. Barton and Mrs A. Burling for their help. This work was supported in part by an M.R.C. Project Grant to M.A.H.S. and a Ford Foundation Grant. S.B.F. is a Beit Memorial Research Fellow.

\section{References}

Amoroso, E.C. \& Perry, J.S. (1975) The existence during gestation of an immunological buffer zone at the interface between maternal and foetal tissues. Phil. Trans. $R$. Soc. B 271, 343-361.

Beer, A.E. \& Billingham, R.E. (1979) Maternal immunological recognition mechanisms during pregnancy. In Maternal Recognition of Pregnancy, (Ciba Fdn Symp. 64), pp. 293-322. Eds J. Elliott \& M. Whelan. Excerpta Medica, Amsterdam.

Beling, C.G., Cederqvist, L.L. \& Fuchs, F. (1976) Demonstration of gonadotropin during the second half of the cycle in women using intrauterine contraception. Am. J. Obstet. Gynec. 125, 855-585.

Dickmann, Z., Dey, S.K. \& Sengupta, J. (1976) A new concept: control of early pregnancy by steroid hormones originating in the preimplantation embryo. Vitams Horm. 34, 215-242.

Enders, A.C. (1971) The fine structure of the blastocyst. In The Biology of the Blastocyst, pp. 71-94. Ed. R. J. Blandau. University of Chicago Press, Chicago.

Heap, R.B., Flint, A.P. \& Gadsby, J.E. (1979) Role of embryonic signals in the establishment of pregnancy. Br. med. Bull. 35, 129-135.

Knobil, E. (1973) On the regulation of the primate corpus luteum. Biol. Reprod. 8, 246-258.
Perry, J.S., Heap, R.B. \& Amoroso, E.C. (1973) Steroid hormone production by pig blastocyst. Nature, Lond. 245, 45-47.

Saxena, B.B., Hasan, S.H., Haour, F. \& SchmidtGollwitzer, M. (1974) Radioreceptor assay of human chorionic gonadotropin: detection of early pregnancy. Science, N.Y. 184, 793-795.

Siiteri, P.K., Febres, F., Clemens, L.E., Chang, R.J., Gondos, B. \& Stiles, D. (1977) Progesterone and maintenance of pregnancy: is progesterone nature's natural immunosuppressant? Ann. N.Y., Acad. Sci. 286, 384-397.

Surani, M.A.H. (1979) Glycoprotein synthesis and inhibition of protein glycosylation of tunicamycin in preimplantation embryos: influence on compaction and trophoblast adhesion. Cell 18, 217-222.

Van Blerkom, J., Chavez, D.J. \& Bell, H. (1979) Molecular and cellular aspects of facultative delayed implantation in the mouse. In Maternal Recognition of Pregnancy (Ciba Fdn Symp. 64), pp. 141-172. Eds J. Elliott \& M. Whelan. Excerpta Medica, Amsterdam.

Wiley, L.D. (1974) Presence of a gonadotrophin on the surface of preimplantation mouse embryos. Nature, Lond. 252, 715-716 